
allemande

48-1 | 2016

Les expériences coloniales allemandes : échanges, transferts, circulations

Friedrich von Lindequist, koloniale Konzentrationslager und transimperiales Lernen

Jonas Kreienbaum

\title{
CpenEdition
}

Journals

Édition électronique

URL : https://journals.openedition.org/allemagne/371

DOI : 10.4000 /allemagne.371

ISSN : 2605-7913

Éditeur

Société d'études allemandes

Édition imprimée

Date de publication : 15 juin 2016

Pagination : 75-88

ISSN : 0035-0974

Référence électronique

Jonas Kreienbaum, „Friedrich von Lindequist, koloniale Konzentrationslager und transimperiales Lernen", Revue d'Allemagne et des pays de langue allemande [Online], 48-1 | 2016, Online erschienen am: 13 Dezember 2017, abgerufen am 18 Mai 2021. URL: http://journals.openedition.org/allemagne/371 ; DOI: https://doi.org/10.4000/allemagne.371 


\section{Friedrich von Lindequist, koloniale Konzentrationslager und transimperiales Lernen}

\section{- Jonas Kreienbaum*}

Um 1900 wurde die Konzentration von weiten Teilen der Zivilbevölkerung in speziellen Lagern oder Zonen zu einer Art Standardmittel kolonialer Militärs bei der Bekämpfung ,aufständischer ' Guerillas. Spanien begann mit der systematischen, groß angelegten reconcentración während des kubanischen Unabhängigkeitskrieges (18951898). Wenig später installierte Großbritannien ein System der concentration camps im Rahmen des Südafrikanischen Krieges (1899-1902), während zeitgleich US-Militärs auf den Philippinen mit sogenannten zones of concentration experimentierten. Schließlich errichtete das Deutsche Reich Konzentrationslager zur Beendigung des Krieges mit Herero und Nama in Deutsch-Südwestafrika (1904-1908).

Historikerinnen und Historiker vertreten unterschiedliche Meinungen, wie sich das gehäufte Auftreten dieser Fälle von Bevölkerungskonzentration erklären lässt. Zahlreiche Autorinnen und Autoren nehmen an, ein Lernprozess habe stattgefunden, Briten und Amerikaner hätten sich am spanischen Beispiel und Deutsche am britischen Vorbild in Südafrika orientiert. Eine empirische Begründung dieser Einschätzung liefern sie jedoch kaum ${ }^{(1)}$. Einen entgegengesetzten Erklärungsansatz verfolgt der britische Militärhistoriker Ian F. W. Beckett, dem sich jüngst auch Andreas Stucki angeschlossen hat. Für beide Autoren ist das vermehrte Auftreten von Bevölkerungskonzentrationen in den genannten Kolonialkriegen vor allem die naheliegende Antwort auf ein gemeinsames strukturelles Problem: die Guerilla-Taktik der kolonisierten Gegner, die ihre Stärke vor allem aus der Unterstützung der lokalen Zivilbevölkerung ziehen. Mit

* Wissenschaftlicher Mitarbeiter, Historisches Institut der Universität Rostock.

1 Siehe u.a. Casper W. Erichsen, "The angel of death had descended violently among them". Concentration camps and prisoner-of-war in Namibia, 1904-1908, Leiden, African Studies Centre, 2005, S. 1; Isabel V. Hull, Absolute Destruction. Military Culture and the Practices of War in Imperial Germany, Ithaca, Cornell University Press, 2005, S. 73; Andrzej J. KAminski, Konzentrationslager 1896 bis heute. Geschichte, Funktion, Typologie, München/Zürich, Pieper, 1990, S. 35. 
dieser Konstellation konfrontiert, hätten Militärs unabhängig voneinander ähnliche Lösungsstrategien entwickelt ${ }^{(2)}$. Dieser Aufsatz stellt die Frage nach einem möglichen Wissenstransfer mit Blick auf Süd- und Deutsch-Südwestafrika: Übernahmen deutsche Kolonialbeamte oder -militärs die Idee des Konzentrationslagers aus dem benachbarten britischen Südafrika? Um diese Frage zu beantworten, wird zunächst die Funktion der südafrikanischen Lager und ihre Rezeption im Deutschen Reich untersucht. Anschließend rücken die Konzentrationslager in Südwestafrika und vor allem der Entscheidungsprozess, der zu ihrer Errichtung führte, in den Vordergrund, um abschließend - auf dieser Grundlage - die eigentliche Transferfrage zu adressieren. In den Fokus der Ausführungen tritt dabei ein Akteur, der sich als deutscher Generalkonsul in Kapstadt und späterer Gouverneur in Deutsch-Südwestafrika in einer idealen Position befand, um einen Transfer des Lager-Gedankens entscheidend zu beeinflussen: Friedrich von Lindequist.

\section{Die concentration camps in Südafrika und ihre Rezeption in Deutschland}

Die concentration camps in Südafrika entstanden im Rahmen des Südafrikanischen Krieges, der noch immer häufig unter dem Titel ,Burenkrieg` firmiert. Anders als viele britische Zeitgenossen vermutet hatten, sollte diese kriegerische Auseinandersetzung nicht der kurze „tea time war“ werden, der bis Weihnachten beendet wäre. Vielmehr zog sich der Kampf zwischen dem Britischen Empire und den beiden unabhängigen Burenrepubliken des Transvaal und des Oranje Freistaats, der am 11. Oktober 1899 begann, in drei Phasen bis Mitte 1902. Nachdem die Briten in den ersten Kriegswochen empfindliche Niederlagen einstecken mussten, gelang es den daraufhin deutlich verstärkten britischen Truppen unter dem Oberkommando von Lord Frederick Sleigh Roberts bis Juni 1900, die Burenrepubliken zu überrennen und die beiden Hauptstädte - Pretoria und Bloemfontein - einzunehmen. Mit der Annexion der Republiken schien der Krieg vorbei, doch begann jetzt lediglich ein neuer, langwieriger Abschnitt: der des Guerillakriegs. Die Burenkommandos operierten nun in kleinen Gruppen, die offene Feldschlachten vermieden und stattdessen isolierte feindliche Einheiten und die britischen Nachschublinien attackierten ${ }^{(3)}$. Lord Roberts beantwortete die neue Burentaktik mit einer ,Strategie der verbrannten Erde‘, die später von seinem Nachfolger Lord Horatio Herbert Kitchener systematisiert wurde. Britische Truppen begannen, das Land zu durchkämmen und alles - von Häusern bis zu Lebensmitteln - zu zerstören, was die burischen Kommandos zur Aufrechterhaltung des Widerstandes benötigten. Teil dieser Strategie war es, alle Bewohner der umkämpften Gebiete - Buren wie Afrikaner - in neu errichtete und bewachte Lager zwangsweise umzusiedeln. Dort waren sie unter Kontrolle und folglich außer Stande, die Guerillas mit Nahrung, Waffen,

2 Ian F. W. Beckett, Modern Insurgencies and Counter-Insurgencies. Guerrillas and Their Opponents since 1750, London/New York, Routledge, 2001, S. 36. Stucki argumentiert ein wenig vorsichtiger, indem er das Strukturargument in den Vordergrund rückt, Wissenstransfers aber als durchaus denkbar, jedoch als bisher nicht nachgewiesen einstuft. Vgl. Andreas Stucki, Aufstand und Zwangsumsiedlung. Die kubanischen Unabhängigkeitskriege 1868-1898, Hamburg, Hamburger Edition, 2012, S. 368-376.

3 Zum Südafrikanischen Krieg siehe Thomas Pakenham, The Boer War, London/Sydney, Macdonald \& Co, 1982; Bill Nasson, The War for South Africa. The Anglo-Boer War 1899-1902, Kapstadt, Tafelberg, 2010; Iain R. Sмith, The Origins of the South African War, 1899-1902, London/New York, Longman, 1996. 
Munition, Informationen oder neuen Rekruten zu versorgen. In diesen sogenannten refugee camps, die seit August 1900 errichtet wurden, befand sich auch eine geringere Anzahl an Buren, die ihre Waffen niedergelegt hatten und freiwillig in die Lager gekommen waren. Sie suchten Schutz vor den burischen Kommandos, die diese handsuppers - auch unter Zwang - zur Wiederaufnahme des Widerstandes gegen die Briten bewegen wollten. In beiden Fällen dienten die Lager einem eindeutig militärischen Ziel. Sie waren integraler Bestandteil der britischen Strategie der Guerillabekämpfung ${ }^{(4)}$.

Separate Lagersysteme wurden im Transvaal und der Orange River Colony, wie der Freistaat nach der Annektierung hieß, errichtet, in denen immer mehr burische Frauen, Kinder und einige Männer, die von den britischen Kolonnen eingewiesen wurden, unterkommen mussten. Bald befanden sich über 100.000 Buren in den Camps. Die britische Lagerverwaltung, die zunächst dem Militär und später zivilen Stellen unterstand, war außer Stande, die Masse der Neuankömmlinge ausreichend zu versorgen. Der Mangel an adäquaten Unterkünften, Kleidung, Nahrung, medizinischer Versorgung, sanitären Einrichtungen und vor allen Dingen eine schwere Masernepidemie führten während des Jahres 1901 zum Massensterben in den Konzentrationslagern: zwischen 25.000 und 30.000 Buren, zum großen Teil Kinder, starben in den Lagern ${ }^{(5)}$. Noch schlimmer sah die Versorgungslage in den separat organisierten Lagern für Afrikaner aus, für die vermutlich ähnliche Internierten- und Todeszahlen zu konstatieren sind ${ }^{(6)}$. Die black camps waren ebenfalls Teil der britischen Anti-GuerillaStrategie, aber sie dienten noch weiteren Zwecken. Zunächst einmal fungierten sie als ,Arbeitskräftereservoir ‘ für das britische Militär, das systematisch männliche Lagerinsassen in Dienst nahm. Darüber hinaus waren sie ein Instrument für eine bestimmte Art des social engineering. Anstatt die Insassen - wie in den Burenlagern - gratis mit Nahrung zu versorgen, ließen die britischen Verantwortlichen sie in und um die Lager Land bestellen. Dies galt als geeignete Methode, ihre landwirtschaftlichen Fähigkeiten zu erhalten, um die ,Eingeborenen' dann auch nach dem Krieg als Handlanger auf den Farmen weißer Siedler einsetzen zu können ${ }^{(7)}$. In den ,weißen ' Lagern nahm das

4 Zur britischen Guerillabekämpfung siehe Stephanus Burridge SpIEs, Methods of Barbarism? Roberts and Kitchener and Civilians in the Boer Republics, January 1900-May 1902, Kapstadt, Human \& Rousseau, 1977; Fransjohan Pretorius, „The fate of the Boer women and children“, in: F. Pretorius (Hg.), Scorched Earth, Kapstadt/Pretoria/Johannesburg, Human \& Rousseau, 2001, S. 36-59.

$5 \mathrm{Zu}$ den Lagern siehe Elizabeth van Heyningen, The Concentration Camps of the Anglo-Boer War. A Social History, Johannesburg, Jacana, 2013; vgl. E. vAN Heyningen, „The Concentration Camps of the South African (Anglo-Boer) War, 1900-1902“, History Compass, 7 (2009), S. 22-43; F. Pretorius, Scorched Earth (Anm. 4); Johannes C. Отто, Die Konsentrasiekampe (1954), Pretoria, Protea Boekhuis, 2005.

6 Die lediglich fragmentarisch überlieferten Berichte zu den black camps lassen eine genaue Bezifferung nicht zu. Peter Warwick nannte die oft zitierte Zahl von 14.154 Toten, wobei er sich aber nur auf die Phase von Juli 1901 bis Mai 1902 bezieht. Peter WArwick, Black People and the South African War 1899-1902, Cambridge, Cambridge University Press, 1983, S. 145 u. 151; vgl. zu den Todeszahlen auch Stowell KessLer, „The black concentration camps of the Anglo-Boer War 1899-1902, Shifting the paradigm from sole martyrdom to mutual suffering“, Historia, 44/1 (1999), S. 110-147.

7 Zu den ,schwarzen' Lagern siehe Stowell V. Kessler, The Black Concentration Camps of the Anglo-Boer War, 1899-1902, Bloemfontein, Anglo-Boer War Museum, 2012; B. E. Mongalo, Kobus DuPisani, „Victims of a white man's war, Blacks in concentration camps during the South African War (18991902)“, Historia, 44/1 (1999), S. 148-182; zu den Zielen, die durch die Lager für Afrikaner erreicht 
social engineering andere Formen an. Die Buren sollten zu modernen Subjekten der britischen Krone erzogen werden, wobei das Hauptmittel zur Erreichung dieses Ziels die Schulen waren, die in allen Lagern errichtet wurden. Der Unterricht fand - mit Ausnahme der Religionsstunden - auf Englisch statt, um so die Masse der Burenkinder zu anglisieren ${ }^{(8)}$.

Die miserable Versorgungslage und das Massensterben in den Burenlagern führte Mitte 1901, nachdem Emily Hobhouse einen Bericht über ihre Besuche in zahlreichen südafrikanischen Lagern veröffentlichte, zu einem Aufschrei in der britischen Öffentlichkeit, der auch in anderen Ländern - nicht zuletzt dem Deutschen Reich wiederhallte $^{(9)}$. Der Südafrikanische Krieg war auch in Deutschland bereits zuvor ein Medienereignis und die Zeitungen berichteten täglich darüber. Von nun an aber wurden die Lager für burische Frauen und Kinder eines der prominentesten Themen ${ }^{(10)}$. Auch auf dem Buchmarkt hatte der ,Burenkrieg' Hochkonjunktur. Eine aktuelle Bibliografie zählt allein bis 1910358 deutschsprachige Publikationen zum Krieg in Südafrika ${ }^{(1)}$. Darunter waren Auszüge aus den offiziellen britischen Blaubüchern zu den Lagern, Emily Hobhouses bereits erwähnter Bericht und Elizabeth Neethlings Darstellung der Leiden der burischen Deportierten in den Lagern ${ }^{(12)}$. Pro-burische Vereine wurden gegründet, Spenden gesammelt und als Hilfspakete für die Frauen und Kinder in den concentration camps nach Südafrika gesandt ${ }^{(13)}$.

Diese Entwicklung machte sich auch für Friedrich von Lindequist (1862-1945), der zwischen 1900 und 1902 das Generalkonsulat in Kapstadt leitete, bemerkbar. Lindequist war als studierter Jurist 1892 in den Dienst der Kolonialabteilung des Auswärtigen

werden sollten, siehe Jonas Kreienbaum, „Ein trauriges Fiasko“. Koloniale Konzentrationslager im südlichen Afrika, 1900-1908, Hamburg, Hamburger Edition, 2015, S. 102-110.

8 Elizabeth van Heyningen, „A Tool for Modernisation? The Boer Concentration Camps of the South African War, 1900-1902“, South African Journal of Science, 106/5-6 (2010), Artikel 242. Van Heyningen wurde scharf dafür kritisiert, die Lager als „tools for modernization“ zu beschreiben - vor allem von Fransjohan Pretorius, „The white concentration camps of the Anglo-Boer War, A debate without end“, Historia, 55/2 (2010), S. 34-49; zu den Lager-Schulen siehe Eliza RiedI, „Teaching Empire, British and Dominions Women Teachers in the South African War Concentration Camps", English Historical Review, CXX489 (2005), S. 1316-1347 und Paul Zietsman, „The concentration camp schools - beacons of light in the darkness“, in: F. Pretorius, Scorched Earth (Anm. 4), S. 86-109.

9 Emily Новноuse, Report of a Visit to the Camps of Women and Children in the Cape and Orange River Colonies, London, Friars Printing Association, 1901.

10 Vgl. Steffen Bender, Der Burenkrieg und die deutschsprachige Presse. Wahrnehmungen und Deutungen zwischen Bureneuphorie und Anglophobie 1899-1902, Paderborn u.a., Ferdinand Schöningh, 2009, S. 13-23.

11 Nicol Stassen, Ulrich van der Heyden, German Publications on the Anglo-Boer War, Pretoria, Protea Boekhuis, 2007.

12 O.A., Die Concentrationslager im Transvaal und Orange River Colonie, London, Aug. Siegle, 1902; Emily Новноuse, Die Zustände in den südafrikanischen Konzentrationslagern. Bericht von Miss E. Hobhouse, Berlin, Deutscher Burenhilfsbund, 1902; Elizabeth NeEthling, Fünfzehn Monate in den Konzentrationslagern. Erinnerungen einer Burenfrau aus ihrer Gefangenschaft, Bern, Berner Tagblatt, 1903.

13 Ulrich KRöLL, Die internationale Buren-Agitation 1899-1902. Haltung der Öffentlichkeit und Agitation zugunsten der Buren in Deutschland, Frankreich und den Niederlanden während des Burenkrieges, Münster, Regensberg, 1973. 
Amtes getreten und 1894 nach Deutsch-Südwestafrika entsandt worden, wo er bereits zwei Jahre später zum ständigen Vertreter des Landeshauptmanns Theodor Leutwein aufstieg. Nach seiner Zeit in Kapstadt kehrte er in die Kolonialabteilung nach Berlin zurück, um von 1905 bis 1907 das Amt des Gouverneurs in Südwestafrika und schließlich ab 1910 die Nachfolge von Bernhard Dernburg als Staatssekretär und damit Leiter des neu geschaffenen Reichskolonialamts anzutreten ${ }^{(14)}$. Als Generalkonsul in Kapstadt kam er auf Grund der Spendenflut aus Deutschland nun in verstärkten Kontakt mit den concentration camps, da er an der Distribution der deutschen "Liebesgaben“ in den Lagern beteiligt war ${ }^{(15)}$. Jahrzehnte später hielt er fest: „Bei meinen Besuchen der größten [sic] Frauen- und Kinder-Lager in Bloemfontein und Johannesburg, konnte ich mich von dem richtigen Eintreffen und der Nichtbeanstandung der Gaben, die mit durch die Hände unserer dortigen deutschen Pfarrer gingen, überzeugen “(16). In seinem ausführlichen Bericht über den Besuch der beiden Lager an Reichskanzler Bernhard von Bülow schilderte er seinen Eindruck, der „kein sehr günstiger“ war. Der Zustand in den „refugee camps“ unterscheide sich, was die persönliche Freiheit angehe, „,von dem der Kriegsgefangenschaft sehr wenig“. Weiter vermerkte er:

„Die Unterbringung ist naturgemäß eng für die meist sehr zahlreichen Familien. Die Wellblechbaracken oder kleinen Zelte bieten den ungenügend gekleideten refugees keinen hinreichenden Schutz gegen die scharfe Kälte. Die Ernährung war in Johannesburg anfangs sehr schlecht und viel zu knapp. Doch ist hierin mit dem Wechsel des Oberaufsehers eine wesentliche Besserung eingetreten. Die Sterblichkeit, welche an sich schon sehr groß war, ist durch in den Camps ausgebrochene Masernepidemien noch sehr erhöht worden, sodaß sie in der Woche auf 100 und mehr Fälle unter 3000 bis 4000 Seelen stieg“(17).

Bereits in einem früheren Bericht nach Berlin hatte er die Lager als Teil der britischen Anti-Guerilla-Strategie identifiziert, deren Ansatz es sei, „das Land von allem, was den Buren möglicher Weise als Hilfsquelle dienen könnte, vor allem von Vieh und Nahrungsmitteln zu entblößen“(18). In Deutsch-Südwestafrika sollten sich die Lager, an deren Errichtung und Management Lindequist entscheidend beteiligt war, in eine andere Strategie einfügen. Die Konzepte aus Südafrika ließen sich nicht eins zu eins übertragen.

14 Zur Vita Lindequists siehe Horst GrüNDER, „Lindequist, Friedrich von“, in: Neue Deutsche Biographie, 14 (1985), S. 601, http,//www.deutsche-biographie.de/pnd117025259.html; „Lindequist, Friedrich v.“, in: Heinrich Schnee (Hg.), Deutsches Kolonial-Lexikon, Bd. 2, Leipzig, Quelle \& Meyer, 1920, S. 456f; Helmut BLEY, Kolonialherrschaft und Sozialstruktur in Deutsch-Südwestafrika 1894-1914, Hamburg, Leibniz-Verlag, 1968, S. 321.

15 Siehe Politisches Archiv des Auswärtigen Amtes (PA AA), Kapstadt 54. Bi., „Liebesgaben für die Konzentrationslager“, 1899-1904.

16 Manuskript, „Generalkonsul des Deutschen Reiches für Britisch-Südafrika in Kapstadt“, Bundesarchiv Koblenz (BAK), N 1669, Nachlaß Friedrich von Lindequist (1895-1903); vgl. auch National Archives London (NAL), Colonial Office (CO) 417 South Africa /348 Bd. VI. Miscellaneous. Despatches, January 1902, B1. 1021-1024. Bloemfontein und Johannesburg waren keinesfalls die größten Camps.

17 Friedrich von Lindequist an Bernhard von Bülow, Kapstadt, den 23.6.1901, Nr. 2563, PA AA, Kapstadt 34. Politische Angelegenheiten B1, März 1901 bis Juli 1901.

18 Friedrich von Lindequist an Bernhard von Bülow, Kapstadt, den 16.6.1901, Nr. 2464, ebd.; vgl. auch G. an Bernhard von Bülow, Kapstadt den 29.4.1901, Nr. 1763, ebd. 


\section{Die Entscheidung zur Errichtung von Konzentrationslagern in Deutsch-Südwestafrika}

Wie in Südafrika gehörten Konzentrationslager zu Beginn des Krieges, der am 12. Januar 1904 im deutschen, Schutzgebiet' ausbrach, noch nicht zum Repertoire des Kolonialmilitärs. Erst nachdem der deutsche Kommandeur, Generalleutnant Lothar von Trotha, die gegnerischen Herero am Waterberg geschlagen, sie anschließend erfolglos in die Omaheke-Wüste verfolgt und schließlich das Sandfeld von seinen Truppen hatte abriegeln lassen, kam es zur Einrichtung der Lager ${ }^{(19)}$. Trotha hatte im Zuge der Abriegelung seinen berüchtigten ,Vernichtungsbefehl' vom 2. Oktober 1904 erlassen, der das Machen von Gefangenen verbot. Reichskanzler Bülow und Generalstabschef Alfred von Schlieffen sprachen sich gegen diesen „Genozid-Befehl“(20) aus und erreichten beim Kaiser Anfang Dezember dessen Aufhebung. Im Zuge dieser Änderung der Kriegsstrategie ordnete Bülow nun gegenüber Trotha an, „Konzentrationslager für die einstweilige Unterbringung \& Unterhaltung der Reste des Herero-Volkes“ zu errichten ${ }^{(21)}$. In diese Lager, die in den Küstenorten Swakopmund und Lüderitzbucht und im Inland in Karibib, Okahandja, Windhuk und Omaruru errichtet wurden und die bis 1908 insgesamt über 20.000 Insassen beherbergten, wurden seit Ende 1905 auch zunehmend Nama gebracht. Die vornehmlich im Süden der Kolonie lebenden Nama hatten erst im Oktober 1904 die Waffen gegen die Kolonialmacht erhoben. Anders als die Herero im Norden, die nach der Schlacht am Waterberg militärisch praktisch geschlagen waren, stellten die als Guerillas kämpfenden Nama bis Ende 1906 eine ernsthafte militärische Gefahr für die Deutschen dar.

Wie in Südafrika war der Primärzweck der "Gefangenenkraale "ein militärischer: Sie sollten zur Beendigung eines langwierigen Kolonialkriegs beitragen. Dennoch unterschied sich ihre Logik signifikant. Ging es in Südafrika um die effektive Trennung der Kommandos von der sie unterstützenden Zivilbevölkerung, indem die Zivilisten flächendeckend in Lager deportiert und dort kontrolliert wurden, machte ein solches Vorgehen in Südwestafrika keinen Sinn. Die Herero kämpften nicht als Guerillas und die Nama, die sich tatsächlich der Methoden des kleinen Krieges bedienten, waren zu ihrer Versorgung nicht auf eine sesshafte Bevölkerung angewiesen. Sie versorgten sich durch Überfälle auf deutsche Nachschubkolonnen und bei Händlern im unübersichtlichen Grenzgebiet zwischen der britischen Kapkolonie und dem deutschen ,Schutzgebiet'. Ihre Familien begleiteten sie entweder im Feld oder sie traten im Laufe des Krieges auf englisches Gebiet über. In beiden Fällen gab es keine sesshafte

19 Zum Kriegsverlauf und vor allem zur Radikalisierung der Kriegsführung siehe I. Hull, Absolute Destruction (Anm. 1), S. 5-69; Matthias HÄUssler, „From Destruction to Extermination, Genocidal Escalation in Germany's War Against the Herero“ (1904), Journal of Namibian Studies, 10 (2011), S. 55-81; Horst Drechsler, Südwestafrika unter deutscher Kolonialherrschaft. Der Kampf der Herero und Nama gegen den deutschen Imperialismus (1884-1915), Berlin, Akademie-Verlag, 1984 (2. Aufl.).

20 Horst Gründer, Geschichte der deutschen Kolonien, Paderborn u.a., Ferdinand Schöningh, 2004 (5. Aufl.), S. 122.

21 Tel. Bernhard von Bülow an Lothar von Trotha, Berlin, den 11.12.1904, Bundesarchiv-Berlin (BAB), Reichskolonialamt (R 1001)/2089 Differenzen zwischen Generalleutnant Lothar v. Trotha und Gouverneur Theodor Gotthilf Leutwein über das Verhältnis von militärischen und politischen Maßnahmen zur Beendigung des Krieges, Bl. 54. 
Zivilbevölkerung, die nach südafrikanischem Vorbild von den deutschen Militärs hätte zwangsumgesiedelt werden können ${ }^{(22)}$. In die deutschen Lager kamen stattdessen jene Herero und Nama, die gefangen genommen wurden oder die sich freiwillig - vor allem auf den Sammelstationen der Rheinischen Mission - stellten. Die Internierung diente nun dazu, zu verhindern, dass die genannten Personen zurück ins Feld gingen und sich erneut den ,Aufständischen' anschlossen. Die Lager erinnerten in dieser Hinsicht durchaus an klassische Kriegsgefangenenlager und tatsächlich wurden die Internierten - auch Frauen und Kinder - offiziell als Kriegsgefangene bezeichnet. Hinzu kam der Wunsch, die Arbeitskraft der Gefangenen - durch Zwangsarbeit auszubeuten, da sowohl die Kolonialwirtschaft als auch das Kolonialmilitär während des Krieges unter massivem Arbeitskräftemangel litten. Damit verquickt war das Ziel, die Internierten eben durch diese Zwangsarbeit zur Arbeit zu erziehen und so für die Nachkriegszeit ein fügsames Arbeiterproletariat zu schaffen. Schließlich galt die „Leidenszeit“(23) in den Lagern als gerechte Strafe für den ,Aufstand ${ }^{‘(24)}$. Eine Konsequenz dieser Zielsetzungen und vor allem der massiven logistischen Probleme und des generellen Desinteresses an Gefangenenfragen war die mangelhafte Versorgung der Internierten, die wie in Südafrika zum Massensterben in den Lagern führte: Laut offizieller Zahlen starben 7682 der gefangenen Herero und Nama ${ }^{(25)}$.

Woher kam nun aber im Dezember 1904 die Idee, Konzentrationslager in DeutschSüdwestafrika zu etablieren und welche Rolle spielte dabei das südafrikanische, Vorbild'? Wie bereits oben ausgeführt, war der Südafrikanische Krieg ein Medienereignis im Deutschen Reich gewesen, wobei gerade die concentration camps eine prominente Rolle gespielt hatten. 1904 war den deutschen Akteuren in Berlin wie in Südwestafrika der britische ,Präzedenzfall' mit Sicherheit bekannt und tatsächlich diente er mitunter als Referenz, um die Ereignisse in der eigenen Kolonie einzuordnen. In diesem Sinne schrieb Paul Leutwein, Sohn des früheren Gouverneurs Theodor Leutwein, über Trothas Kriegsführung: „Er konnte auch auf das Beispiel Kitcheners überweisen, der vor wenigen Jahren Konzentrationslager für die Frauen und Kinder der Buren eingerichtet hatte, um dieses [sic], um seine Selbstständigkeit ringende Volk zur Ergebung zu zwingen“(26).

Doch stammte das Wissen über die britischen Lager nicht allein aus den Medien, wie das Beispiel Lindequist zeigt. Neben Lindequist gab es noch weitere deutsche Beamte und Offiziere, die in Südafrika selbst mit der, Materie‘ in Berührung gekommen waren

22 Vgl. dazu ausführlich Jonas Kreienbaum, „Guerrilla Wars and Colonial Concentration Camps. The Exceptional Case of German South West Africa (1904-1908)“, Journal of Namibian Studies, 11 (2012), S. 85-103.

23 Hans Tecklenburg an Kolonialabteilung, Windhuk den 2.7.1905, R 1001/2118 Aufstand der Hereros 1904-1907, Bd. 8, März 1905-Jan. 1906, Bl. 154f.

$24 \mathrm{Zu}$ den Zwecken, die die Lager in Südwestafrika erfüllen sollten, siehe ausführlich J. Kreienbaum, „Ein trauriges Fiasko“ (Anm. 7), S. 86-120.

25 Sterblichkeit in den Kriegsgefangenenlagern in Südwestafrika, R 1001/2140 Aufstand im Namaland (Namaqualand) und seine Bekämpfung, Bd. 8, Febr. 1907-Febr. 1909, Bl. 161f. Zur Diskussion der Zahl siehe I. Hull, Absolute Destruction (Anm. 1), S. 89f.

26 BAK, N 1145 Nachlaß Paul Leutwein (1882-1946), Bd. 4, Manuskript, „Im Banne Afrikas. Romantisches Geschichtsbild des alten Wild-Südwestafrikas“. 
und nun mitunter zentrale Rollen bei der Errichtung und Gestaltung der Konzentrationslager in Südwestafrika spielten. Zu verweisen ist in diesem Kontext vor allem auf Ludwig von Estorff, der Mitte 1901 in Südafrika nicht nur die Truppen Walter Kitcheners - Bruder des Oberbefehlshabers - während des Guerillakrieges für einige Wochen begleitet, sondern auch eines der Konzentrationslager zusammen mit seinem Freund Lindequist besichtigt hatte ${ }^{(27)}$. Im April 1907 sollte er, der schon lange in Südwestafrika war, den Oberbefehl über die dortige ,Schutztruppe übernehmen und als eine seiner ersten Amtshandlungen das berüchtigte Lager auf der Haifischinsel räumen lassen. Darüber geriet er in Streit mit Vizegouverneur Oskar Hintrager, dem das als ein unvertretbares Sicherheitsrisiko erschien ${ }^{(28)}$. Hintrager war der dritte hochrangige Offizielle im deutschen ,Schutzgebiet', der direkte Erfahrungen aus dem Südafrikanischen Krieg vorweisen konnte. Er hatte auf Seiten der Buren als Freiwilliger an den Kampfhandlungen teilgenommen ${ }^{(29)}$. Zwar bekam er selbst keins der concentration camps zu Gesicht, schließlich schiffte er sich im September 1900 - als gerade die ersten Lager errichtet wurden - bereits wieder nach Deutschland ein. Doch er blieb auch nach seiner Rückkehr am Schicksal der Buren interessiert, war aktives Mitglied eines pro-burischen Vereins in München, der auch für die internierten Frauen und Kinder sammelte, und sicherlich gut über die Camps informiert ${ }^{(30)}$.

Der erste Vorschlag zur Errichtung von Konzentrationslagern in der deutschen Kolonie stammte dann aber nicht von einem dieser Kolonialbeamten bzw. -militärs und auch nicht von Graf Georg von Stillfried und Rattonitz, wie mitunter behauptet wird, sondern - zumindest soweit die Akten Auskunft geben - von der Rheinischen Mission ${ }^{(31)}$. Missions-Inspektor Gottlob Haussleiter schrieb am 25. November 1904 an den Reichskanzler, bot zum wiederholten Mal die Hilfe der Mission bei der Vermittlung des Friedens im ,Schutzgebiet' an und fügte einen neuen Vorschlag hinzu:

„Endlich möchten wir uns gestatten, die alsbaldige Bezeichnung von Freistätten in Anregung zu bringen, an welchen diejenigen Herero, die sich zwar am Krieg, aber nicht am Meuchelmord beteiligt haben, unter Niederlegung ihrer Waffen Schonung des Lebens und

27 Ludwig von EstorfF, Wanderungen und Kämpfe in Südwestafrika, Ostafrika und Südafrika 18941910, Wiesbaden, Kutscher, 1968, S. 93 f.

28 Ausführlich zum Konflikt um die Räumung der Haifischinsel siehe J. Kreienbaum, „Ein trauriges Fiasko" (Anm. 7), S. 128-132.

29 Siehe biographische Angaben zu Hintrager, BAK, N 1037 Nachlaß Oskar Hintrager.

30 Vgl. J. J. Овеrholster, „Inleiding“, in: Christiaan de Wet-Annale, 2 (1973), S. 5-10, hier S. 6. und Oskar Hintrager, Steijn, de Wet und die Oranje-Freistaater. Tagebuchblätter aus dem südafrikanischen Kriege, Tübingen, Laupp, 1902.

31 David Olusoga, Casper W. Erichsen, The Kaiser's Holocaust. Germany's Forgotten Genocide and the Colonial Roots of Nazism, London, Faber and Faber, 2010. Stillfried spricht in seinem Bericht an den Kaiser von „geschlossenen Niederlassungen“ nahe den Arbeitsplätzen, an denen die ,Eingeborenen in Zukunft unterzubringen seien. Dass es sich dabei um Konzentrationslager und nicht etwa um Dörfer oder ,Lokationen', die es bereits zu Friedenszeiten im südlichen Afrika gab, handeln soll, ist Spekulation. Vor allem aber ist der Bericht auf den 12. Dezember 1904 datiert, also einen Tag, nachdem der erste Befehl zur Errichtung von Konzentrationslagern bereits erteilt war, und kann folglich nicht ,Ursprung' der Konzentrationspolitik sein. Bericht des Oberleutnants Graf von Stillfried und Rattonitz betreffend Eingeborenenfrage und militärische Verhältnisse in SWA i.d. letzten zwei Jahren, Berlin, den 12.12.1904, R 1001/2117 Aufstand der Hereros 1904-1907, Bd. 7, Dez. 1904-März 1905, Bl. $59 f$. 
Aufnahme finden könnten. [...] Als solche Freistätten könnten zunächst einzelne Wasserstellen bezeichnet werden, in deren Nähe Konzentrationslager errichtet würden [... “(32).

Dem Reichskanzler war das Konzept des Konzentrationslagers zu diesem Zeitpunkt sicherlich nicht fremd und das lag nicht allein an der intensiven Berichterstattung in den Medien. Lindequist und andere Diplomaten hatten ihn während des Südafrikanischen Krieges mit teils detaillierten Berichten versorgt ${ }^{(33)}$. Auch waren ihm die ausführlichen britischen Blaubücher, die ein differenziertes Bild der concentration camps und ihrer Funktionsweise zeichneten, zugänglich ${ }^{(34)}$. Bülow nahm den Vorschlag der Mission umgehend auf und wies Trotha am 11. Dezember im Zuge der Aufhebung des Vernichtungsbefehls an, Konzentrationslager zu schaffen ${ }^{(35)}$. Wenig später bestätigte Bülow die Order und ergänzte, dass die Herero in den Lagern unter Bewachung zur Arbeit angehalten werden sollten. Am Rand des Schriftstückes befindet sich eine handschriftliche Notiz, dass „Generalkonsul Lindequist ganz damit einverstanden“" sei ${ }^{(36)}$. Als Lindequist im November 1905 dann seinen Dienst als Gouverneur in Südwestafrika antrat, war es eben die Arbeitskraftausbeutung der Internierten, die er zunehmend in den Vordergrund rückte, wodurch er das Lagersystem insgesamt spürbar veränderte. Durch die Gewährung geringer Löhne und die Kooperation mit der Rheinischen Mission suchte er mehr Herero in die Lager zu locken und diese dann zu besserer Arbeitsleistung zu motivieren. Gerade in der Einführung von Löhnen, die sein Vorgänger Trotha noch strikt abgelehnt hatte, lässt sich ein gewisser Schritt in Richtung der britischen Lagerorganisation sehen. Dort wurde Arbeit - mit Ausnahme von gewissen Diensten zur Aufrechterhaltung der Lagerinfrastruktur - grundsätzlich entlohnt. Das Maß an Zwang, das zur Arbeitskraftausbeutung verwendet wurde, blieb in den deutschen Lagern aber weiterhin deutlich höher als in den britischen Camps ${ }^{(37)}$.

Lindequist, der direkte Erfahrungen mit den südafrikanischen Lagern gemacht hatte, war also unmittelbar an der Entscheidung, Konzentrationslager in Südwestafrika einzurichten, beteiligt. Eine besondere Verbindung zu Südafrika hatte übrigens auch die Rheinische Mission. Ihr Missionsgebiet erstreckte sich im südlichen Afrika bis auf das Gebiet der Kapkolonie, und so waren auch Rheinische Missionare vom Südafrikanischen Krieg direkt betroffen, wenn sie auch - anders als viele ihrer

32 Gottlob Haussleiter an Bernhard von Bülow, Barmen den 25.11.1904, Archives of the EvangelicalLutheran Church in the Republic of Namibia (ELCRN), II. Innere Verwaltung 5.14 Deputation. Verschiedenes, 1904-1907 (keine Blattzählung).

33 Siehe etwa Lindequist an Bülow, 23.6.1901, Nr. 2563, Kapstadt 34 B1. G. an Bülow, 29.4.1901, Nr. 1763, ebd. Konsul Pretoria an Bernhard von Bülow, Pretoria, den 5.12.01, Nr. 13912, PA AA, AI Afrika. Den Krieg Englands gegen Transvaal und den Oranjefreistaat, 23.8.1901 bis 17.10.1901, Bd. 41.

34 Exemplare der wichtigen Blaubücher Cd. 819, Cd. 853, Cd. 902 finden sich in den Akten des Auswärtigen Amtes, dem Bülow vorstand. Siehe PA AA, R 14758-14760, AI Afrika. Den Krieg Englands gegen Transvaal und den Oranjefreistaat, 18.10.1901 bis 7.2.1902, Bd. 42-44.

35 Bülow an Trotha, 11.12.1904, R 1001/2089, Bl. 54.

36 Bernhard von Bülow an Lothar von Trotha, Berlin, den 13.1.1905, ebd., Bl. 116f.

37 Ausführlich zur Arbeit in den südwestafrikanischen Lagern siehe Jonas Kreienbaum, „,Wir sind keine Sklavenhalter ${ }^{`}$ - Zur Rolle der Zwangsarbeit in den Konzentrationslagern in Deutsch-Südwestafrika (1904-1908)“, in: Christoph JAHr, Jens Thiel (Hg.), Lager vor Auschwitz. Gewalt und Integration im 20. Jahrhundert, Berlin, Metropol, 2013, S. 68-83. 
Kollegen von der Hermannsburger und Berliner Mission - nicht selbst interniert worden waren ${ }^{(38)}$. Eine intensive Rezeption der südafrikanischen Lager durch die Rheinische Mission lässt sich aber aus den Aktenbeständen ersehen ${ }^{(39)}$.

Natürlich ist die Partizipation Lindequists an der Entscheidungsfindung noch kein Beweis, dass das Konzept des Konzentrationslagers in einem Prozess des Wissenstransfers aus der britischen Nachbarkolonie übermittelt wurde. Einige weitere Überlegungen lassen diese Annahme aber als außerordentlich wahrscheinlich erscheinen.

Zunächst einmal war es im Deutschen Reich üblich, die erfahrene britische Kolonialmacht als Vorbild zu sehen. Ulrike Lindner geht in ihrer exzellenten Studie zu den „Kolonialen Begegnungen“ Deutschlands und Englands in Afrika davon aus, dass "Großbritannien stets als Bezugspunkt kolonialer Überlegungen“ diente, als „[...] das Vorbild, an dem sich die Deutschen abarbeiteten, nachahmend und abgrenzend“(40). Ein zeitgenössischer Autor forderte in diesem Sinne: „Ich möchte es als ein Haupterforderniß [sic] erklären, daß jeder Beamte in unserem Schutzgebiet, der berufen ist, eine maßgebende Stelle daselbst einzunehmen, erst nach Südafrika geschickt werde, um die dortigen Verhältnisse zu studieren und zu sehen, wie man es machen und wie man es nicht machen soll“(41). Und in militärischen Fachjournalen wurde ganz konkret nach den Lehren gefragt, die das Reich aus dem ,Burenkrieg' ziehen könne ${ }^{(42)}$.

Vor allem ist aber darauf zu verweisen, dass das Wort ,Konzentrationslager erst mit dem Südafrikanischen Krieg Einzug in die deutsche Sprache erhielt. Deutsche Konversationslexika führten den Begriff um die Jahrhundertwende noch nicht. Erst in den 1920er-Jahren tauchte er auf, wobei er in der Regel eng mit den Burenlagern verknüpft wurde ${ }^{(43)}$. In der deutschsprachigen Tagespresse firmierten die südafrikanischen Lager zunächst eher als „Frauenlager“ oder „Flüchtlingslager“, und erst allmählich - wie auch in der britischen Presse - setzte sich der Begriff „Konzentrationslager“

38 Siehe die Berichte der Rheinischen Missions-Gesellschaft, 59 (1902). Georg Haccius, Aus der Drangsalszeit des südafrikanischen Lüneburg, Hermannsburg, Verl. der Missionshandlung, (verm. 1904); vgl. auch Ulrich VAN DER HEYdEN, „Der ,Burenkrieg‘ von 1899 bis 1902 und die deutschen Missionsgesellschaften“, in: Ders., Jürgen Becher (Hg.), Mission und Gewalt. Der Umgang christlicher Missionen mit Gewalt und die Ausbreitung des Christentums in Afrika und Asien in der Zeit von 1792 bis 1918/19, Stuttgart, Steiner, 2000, S. 207-223, hier S. 214.

39 Hanns Lessing verweist auf einen entsprechenden Aktenbestand im Missionsarchiv in Wuppertal. Hanns Lessing, „,In der Nähe dieser Wasserstelle sollen Konzentrationslager errichtet werden'. Eine theologische Rekonstruktion der Rolle der Rheinischen Missionsgesellschaft während des Kolonialkrieges in Namibia (1904-1908)“, in: DERs. u.a. (Hg.), Deutsche evangelische Kirche im kolonialen südlichen Afrika. Die Rolle der Auslandsarbeit von den Anfängen bis in die 1920er Jahre, Wiesbaden, Harrassowitz, 2011, S. 471-495, hier S. 493.

40 Ulrike Lindner, Koloniale Begegnungen. Deutschland und Großbritannien als Imperialmächte in Afrika 1880-1914, Frankfurt am Main/New York, Campus, 2011, S. 8, vgl. auch 40f, 52-59, 84-100.

41 Georg Hartmann, Deutsch-Südwestafrika im Zusammenhang mit Südafrika, Berlin, W. Süsserott, 1899, S. 12.

42 Siehe etwa, „Was lehrt uns der Burenkrieg?“, Militär-Wochenblatt, 42 (1902), S. 1146-1147; oder „Die Nutzbarmachung der Erfahrungen des Südafrikanischen Krieges“, Militär-Wochenblatt, 85 (1902), S. 2273f. Dabei ging es jedoch vor allem um Fragen der Infanterie-Taktik und nicht um Anti-GuerillaMaßnahmen, zu denen die Konzentrationslager zählten.

43 Siehe etwa „Konzentrationslager“, in: Meyers Lexikon, Sechster Band Hornberg-Korrektiv, Leipzig, Bibliographisches Institut, 1927 (7. Aufl.), Sp. 1723. 
durch $^{(44)} .1904$ war der Terminus ,Konzentrationslager in Deutschland zweifelsohne unmittelbar mit den Lagern des ,Burenkrieges` verknüpft. Die Tatsache, dass dieser Begriff für die Internierungslager in Südwestafrika von Beginn an und von offizieller Seite verwendet wurde, lässt nur den Schluss zu, dass man sich an den britischen concentration camps orientierte. Anscheinend hatte der britische Kriegsminister St. John Brodrick Recht, als er bezogen auf den Südafrikanischen Krieg behauptet hatte, „die ausländischen Truppen hätten eingesehen, dass sie viel von der englischen Armee lernen könnten“(45).

Mit Blick auf die unterschiedlichen Zwecke ${ }^{(46)}$ der Lager in Süd- und Südwestafrika, die oben skizziert wurden, wird aber sehr deutlich, dass der Prozess des Wissenstransfers notwendig ein „kreativer“ sein musste: „wenig gradlinig, immer brüchig, ziemlich eigensinnig und voller Phantasie“, wie Birthe Kundrus es umschreibt ${ }^{(47)}$. Das System der Burenlager konnte nicht einfach eins zu eins im deutschen, Schutzgebiet' installiert werden, dazu war der Kontext zu verschieden. Die primäre Funktion der Burenlager, den Guerillas durch die Konzentrierung der unterstützenden Zivilbevölkerung die Grundlage zu entziehen, war auf Südwestafrika nicht übertragbar. Die Herero agierten nicht als Guerillas, und die Nama, die tatsächlich die Methoden des Kleinkrieges wählten, waren für ihren Nachschub nicht auf die Zivilbevölkerung angewiesen. Die präventive Trennung von Zivilisten und Kombattanten machte hier keinen Sinn, ebenso wenig wie es die Übernahme der Anglisierungspolitik - die zweite Funktion der Burenlager - gemacht hätte. Insofern war es bei der Rezeption des Konzentrationslager-Konzeptes in Südwestafrika notwendig, dem ,fremden' Import lediglich Bruchstücke zu entnehmen, diese zu bearbeiten und mit Eigenem zu verquicken, wie es Christiane Eisenberg in ihren Überlegungen zum Kulturtransfer formuliert, um ein nutzbares Konzept zu erhalten ${ }^{(48)}$. Übertragbar war nur die vage Idee, eine als feindlich betrachtete Bevölkerung - vor allem auch Frauen und Kinder - als Gefangene an einem umzäunten Ort, in einem Lager, festzuhalten. Das entsprach in etwa dem Bild, welches in der deutschen Öffentlichkeit von den Burenlagern vorherrschte, sofern sie nicht gleich als Vernichtungsstätten verstanden wurden ${ }^{(49)}$. Dass burische Frauen und Kinder offiziell ,Flüchtlinge, und keine Gefangenen waren, und dass die britischen Lager teilweise gar nicht umzäunt waren, spielte, da es im Deutschen Reich kaum wahrgenommen wurde, keine Rolle. Auch intern hatte Lindequist in seinem Bericht über den Besuch in den Lagern von Johannesburg und Bloemfontein an Reichskanzler Bülow betont, dass der Unterschied zu Kriegsgefangenen gering sei ${ }^{(50)}$.

44 Diese Zitate entstammen der Kreuzzeitung, in der erstmals am 24. Juli 1901 von „KonzentrationsLagern“ die Rede ist. Neue Preußische Zeitung, 21.6.1901, 22.6.1901, 24.7.1901, 13.9.1901.

45 „Der Krieg zwischen England und den Buren“, Neue Preußische Zeitung, 6.6.1901. Und auch der Funktionsweisen, auf die in diesem Aufsatz nicht eingegangen werden kann.

Birthe Kundrus, „Kontinuitäten, Parallelen, Rezeptionen. Überlegungen zur ,Kolonialisierung des Nationalsozialismus“, Werkstatt Geschichte, 43 (2006), S. 45-62, hier S. 61.

48 Christiane EisenberG, „Kulturtransfer als historischer Prozess. Ein Beitrag zur Komparatistik“, in: Hartmut Kaelble, Jürgen Schriewer (Hg.), Vergleich und Transfer. Komparatistik in den Sozial-, Geschichts- und Kulturwissenschaften, Frankfurt am Main/New York, Campus, 2003, S. 399-417. 
Die grobe Vorstellung vom Zweck und der Funktion der Konzentrationslager vermischte sich dann vor Ort mit lokalen Einflüssen. Ihre Unterkünfte mussten die gefangenen Herero und Nama in den Lagern selbst errichten. Dazu nutzten sie die vor Ort verfügbaren Materialien, um auf die traditionell übliche Art und Weise sogenannte Pontoks (Rundhütten) zu konstruieren. Die Lager ,umzäunte' das Militär mit Dornbuschzweigen, wie sie im ,Schutzgebiet`sonst auch bei der Einkraalung (Umzäunung) von Rindern genutzt wurden. Und die Verpflichtung der Internierten zur Zwangsarbeit war ebenfalls ein in Südwestafrika erprobtes Mittel zur Bestrafung ,aufständischer' Kolonisierter. So hatte die Kolonialmacht die Zwartboois, eine im Norden des ,Schutzgebietes‘ wohnende Nama-Gruppe, nach ihrem ,Aufstand 'im Jahr 1898 auf diese Weise bestraft ${ }^{(51)}$.

Anders als im Fall der Burenlager sind Gemeinsamkeiten zwischen den Hereround Nama-Lagern und den südafrikanischen black camps leichter auszumachen. Die ähnliche Behausung und die Ausnutzung der Arbeitskraft der Internierten sind zwei ins Auge stechende Charakteristika, die beide Fälle teilen. Doch ist die Existenz von Lagern für Afrikanerinnen und Afrikaner während des Südafrikanischen Krieges von deutscher Seite kaum wahrgenommen worden ${ }^{(52)}$. Ein Transferprozess in dieser Hinsicht scheint daher unwahrscheinlich.

Plausibler ist es, die Parallelen hier als Produkte geteilter Grundannahmen einer gemeinsamen ,kolonialen Kultur' zu verstehen. Zu den Doktrinen dieser Kultur zählten die ,Erziehung' der ,Eingeborenen` zur Arbeit, das Verständnis, ihnen lediglich einen Platz als Lohnarbeitsproletariat im Dienst von Europäern zuzuweisen sowie die Bereitschaft, im Krisenfall nur dann helfend einzugreifen, wenn andernfalls das Wohl der weißen Bevölkerung in Gefahr war. Diese Doktrinen hatten großen Einfluss sowohl in den südwestafrikanischen Gefangenenlagern wie in den südafrikanischen black camps. Sie wirkten jedoch nicht im luftleeren Raum, sondern in Kombination mit strukturellen Faktoren vor Ort. Der Arbeitskräftemangel in Süd- wie Südwestafrika muss als strukturelles Merkmal mit berücksichtigt werden, will man erklären, warum es in beiden Fällen zur flächendeckenden Ausnutzung der Internierten als Arbeitskräfte beim Militär kam. Und die Unterversorgung der internierten ,Eingeborenen' - aber auch der Buren - wurde durch lokale Transportprobleme weiter verschlimmert $^{(53)}$.

51 Vgl. Jürgen Zimmerer, Deutsche Herrschaft über Afrikaner. Staatlicher Machtanspruch und Wirklichkeit im kolonialen Namibia, Hamburg, LIT Verlag, 2001, S. 182.

52 Nur ausnahmsweise wurde die Konzentrierung der afrikanischen Bevölkerung erwähnt, etwa in: „Der Krieg zwischen England und den Buren“, Neue Preußische Zeitung, 5.8.1901.

53 Dieses Erklärungsmodell kann als Synthese aus Isabel Hulls Konzept der „Military Culture“, die den Einfluss von nicht hinterfragten Grundannahmen betont, und Susanne Kuß’ Konzept des „Kriegsschauplatzes“ verstanden werden, das lokale Gegebenheiten für die Entwicklung von Kolonialkriegen hervorhebt. Es ist jedoch zu betonen, dass Hull durchaus lokale Konstellationen mit in ihre Überlegungen einbezieht. Vgl. I. Hull, Absolute Destruction (Anm. 1) und Susanne Kuss, Deutsches Militär auf kolonialen Kriegsschauplätzen. Eskalation und Gewalt zu Beginn des 20. Jahrhunderts, Berlin, Links, 2010, S. 29-37. 


\section{Fazit}

Zusammenfassend kann festgestellt werden, dass die Errichtung der Konzentrationslager in Südwestafrika und ihre konkrete Ausgestaltung ein Produkt verschiedener Einflüsse war. Die britischen concentration camps spielten als Vorbild eine Rolle, wobei Friedrich von Lindequist als Wissensübermittler und später auch als Mitinitiator und Mitgestalter der Lager in Südwestafrika von großer Bedeutung war. Im Zuge der Rezeption wurde das importierte Konzept dann aber strukturellen Gegebenheiten der südwestafrikanischen Kolonialkriegssituation angepasst, vermischte sich mit lokalen Traditionen und wurde durch eine transimperial geteilte ,koloniale Kultur mitgeprägt. Für die Erklärung des gehäuften Auftretens von Bevölkerungskonzentration in Kolonialkriegen um 1900 bedeutet das: Ältere Ansätze, die entweder allein das ,Abschauen' der Kolonialmächte oder die strukturell ähnliche Situation in den Kolonialkriegen als Erklärung für die Politiken der Bevölkerungskonzentration auf Kuba und den Philippinen, in Süd- und Südwestafrika bemühen, sind unterkomplex und können die vielfältigen Gemeinsamkeiten und Unterschiede dieser Fälle nicht hinreichend erklären.

\section{Zusammenfassung}

Um 1900 wurde die Konzentration von weiten Teilen der Zivilbevölkerung in speziellen Lagern oder Zonen zu einer Art Standardmittel kolonialer Militärs bei der Bekämpfung , aufständischer' Guerillas. Der Artikel fragt nach den Gründen für dieses vermehrte Auftreten und richtet dabei den Blick auf mögliche Wissenstransfers zwischen Kolonialimperien. Am Beispiel der Lager, die Großbritannien während des Südafrikanischen Krieges (1899-1902) und das deutsche Kaiserreich im Krieg gegen Herero und Nama (1904-1908) errichten ließen, argumentiert der Text, dass es zu einer Adaption des Lagerkonzepts über koloniale Grenzen hinwegkam. Dabei spielte der ehemalige deutsche Generalkonsul in Kapstadt und spätere Gouverneur von Deutsch-Südwestafrika Friedrich von Lindequist eine entscheidende Rolle. Es ist jedoch zentral, zu betonen, dass der Rezeptionsprozess ein kreativer war, bei dem das importierte Konzept strukturellen Gegebenheiten der südwestafrikanischen Kolonialkriegssituation angepasst wurde, sich mit lokalen Traditionen vermischte und durch eine transimperial geteilte ,koloniale Kultur' mitgeprägt wurde. Ansätze, die ausschließlich von einem Lernprozess oder von strukturellen Parallelen ausgehen, um das gehäufte Auftreten von Konzentrationspolitiken um 1900 zu erklären, sind dagegen unterkomplex und können die vielfältigen Gemeinsamkeiten und Unterschiede dieser Politiken nicht hinreichend erklären.

\section{Résumé}

Vers 1900, la concentration massive de la population civile dans des camps ou des zones spécifiques devint une sorte d'outil standardisé des militaires coloniaux dans la lutte contre les guérillas "rebelles". L'article interroge les causes de ce développement et cherche les éventuels transferts de savoir entre les empires coloniaux. Il étudie les exemples des camps construits par les Britanniques pendant la guerre sud-africaine (1899-1902) et ceux érigés par le Reich pendant la guerre contre les Héréros et les Namas (1904-1908). L'analyse de ces deux exemples révèle une adaptation du concept de camps 
par-delà les frontières coloniales. Friedrich von Lindequist, consul général allemand au Cap, puis gouverneur du Sud-Ouest allemand africain, a joué un rôle décisif dans ce transfert. Le processus de réception de la colonie britannique vers le protectorat allemand s'accompagna d'une transformation. Le concept importé fut adapté aux données structurelles de la situation de guerre coloniale dans le Sud-Ouest africain. Il s'imprégna des traditions locales et fut marqué d'une culture coloniale partagée et transimpériale. Certains modèles d'explication de la multiplication des politiques de concentration vers 1900 présupposent simplement un simple processus d'apprentissage ou des parallélismes structurels. Ils ne reflètent pas la complexité et ne donnent pas d'explication satisfaisante des nombreux points communs ainsi que des différences entre les politiques en question.

\begin{abstract}
At the turn of the 20th century the concentration of civilian populations in special camps or zones somewhat became a model for colonial armies to quell guerrilla resistance. This article investigates the reasons for this development particularly by analysing the possible transfers of knowledge between colonial empires. Focussing on the camps that Great Britain established during the South African War (1899-1902) and those erected by Imperial Germany fighting the Herero and Nama people (1904-1908), this study shows that the concept of camps was extended beyond the colonial boundaries. A key figure in this process was Friedrich von Lindequist, the former German consul general in Cape Town and later governor of German South West Africa. However, it is vital to highlight that the process of reception was a creative one, altering the imported concept to make it fit into the structural conditions of the war in South West Africa, mixing it with local traditions and reshaping it through the influences of a "transimperially" shared 'colonial culture'. Approaches only stressing either processes of learning or structural similarities to explain fin de siècle policies of concentration, lack complexity and are unable to sufficiently account for the multifaceted differences and similarities of these policies.
\end{abstract}

